\title{
Research on Cycle-Related Pseudo-code Parallel Acquisition Algorithm
}

\author{
Yang Liu, Gang Fu \& Dongxu Zhu \\ China Satellite Maritime Tracking and Control Department, Jiangyin, 214431, China
}

ABSTRACT: In the field of measurement and control, spacecraft trajectories are known, in most cases you can use the spacecraft trajectories of spacecraft in terms of the motion brought by the Doppler effect. In the Doppler search range smaller conditions, based on cycle-related pseudo-code parallel acquisition algorithm can reduce the complexity of the algorithm implemented by local preprocessing pseudo-code approach, to some extent, improve the capture rate. And the use of Monte Carlo simulation principles lot Experimental results show the effectiveness and correctness of research ideas and research methods.

KEYWORD: Parallel Acquisition; circular correlation; DSSS

\section{INTRODUCTION}

For traditional serial matched filter correlation peak detection rate of the pseudo-code filters less demanding (M.G.El-tarhhuni, 2012), and have the same data sampling clock can be, but when you want to match the code is very long, the device is consuming resources considerable. Based on pseudo-code domain acquisition folded when matched filtering can save a lot of hardware resources.

\section{DERIVATION ALGORITHM}

To search all of the code phase units within a pseudorandom code period (U. Madhow, 2008), we need to move the local loop pseudo code phase and the reception $\mathrm{PN}$ code correlation, when the phase of the local code and the received code is exactly the same, you have the greatest correlation peak, this cycle in the form of convolution can be expressed as:

$$
s(n)=\sum_{i=0}^{N-1} r(i) c(i+n) \quad n=0,1,2, N-1
$$

Equation (1) may form the following matrix:

$$
\left[\begin{array}{c}
s(0) \\
s(1) \\
\cdots \\
s(N-1)
\end{array}\right]=\left[\begin{array}{cccc}
c(0) & c(1) & \cdots & c(N-1) \\
c(1) & c(2) & \cdots & c(0) \\
\cdots & \cdots & \cdots & \cdots \\
c(N-1) & c(1) & \cdots & c(N-2)
\end{array}\right] \times\left[\begin{array}{c}
r(0) \\
r(1) \\
\cdots \\
r(N-1)
\end{array}\right]=\mathbf{C R}(2)
$$

Equation (2) can capture the time domain pseudocode conversion to the frequency domain using FFT to the pseudo-code phase quick search (Wang Shi lian et al, 2011) (E.G.Strom et al, 2012). The mathematical principle is as follows:

$$
\begin{aligned}
s(n) & =\sum_{i=0}^{N-1} r(i) c(i+n) \\
& =\frac{1}{N} \sum_{k} \sum_{l} R(k) C(-l) \exp \left(j \frac{2 \pi}{N} l k\right) \frac{1}{N} \sum_{i=0}^{N-1} \exp \left[j \frac{2 \pi}{N}(k-l) i\right]
\end{aligned}
$$

Because of

$$
\sum_{i=0}^{N-1} \exp \left[j \frac{2 \pi}{N}(k-l) i\right]= \begin{cases}N & k+l=0, N, 2 N, \cdots \\ 0 & \text { others }\end{cases}
$$

So that equation (4) into:

$$
\begin{aligned}
s(n) & =\frac{1}{N} \sum_{k=0}^{N-1} R(k) C(-k) \exp \left(j \frac{2 \pi}{N} k n\right) \\
& =\operatorname{IFFT}\left\{F F T\left(r(i) \times F F T^{*}(c(i))\right)\right\}
\end{aligned}
$$

Parallel Algorithm Based on Cyclic block diagram related to the pseudo-code as follows:

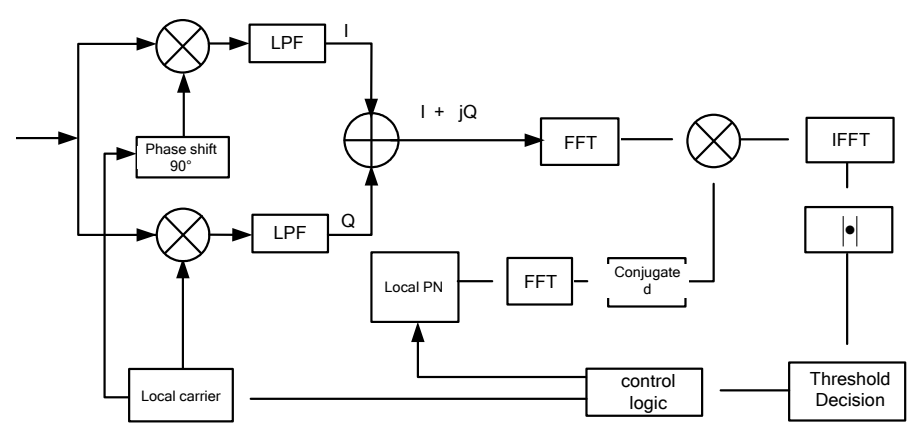

Figure 1. FFT algorithm based on block diagram related cycle 
Its algorithm: Select a carrier frequency uncertainty region, set the value of the carrier frequency in the region, the received signal to do FFT (E.G. Strom et al, 2011), local pseudo-code to do FFT, and to conjugation. Upon receiving the signal and the local PN code sequence multiplying the FFT of the sequence multiplied to do IFFT, inverse transformed sequence modulo searches for the largest peak, if the value does not exceed the threshold value, indicating that the signal is not caught, set frequency step select the next area, repeat the process. If the maximum peak value exceeds the threshold, that signal is captured, the frequency region where the correct time, corresponding to the peak maximum is the correct position of the initial PN code phase value.

\section{CAPTURE PERFORMANCE WITH CARRIER SEARCH STEP}

The signal received by the receiver may be expressed as follows:

$\operatorname{Re}\left(i T_{s}\right)=\sqrt{2 P} D\left(i T_{s}-\tau\right) P N\left(i T_{s}-\tau\right) \cos \left[2 \pi\left(f_{0}+f_{d}\right) i T_{s}+\phi_{0}\right]+n(i)(6)$

Equation (6) fast Fourier transform and inverse transform after the output:

$$
\begin{aligned}
& I(k)=\sqrt{2 P} D(k) R[\rho(k)] \sin c\left[\pi T \Delta f_{d}(k)\right] \cos \left[\Delta f_{d}(k) k T+\varphi_{0}\right]+\tilde{n}_{I}(k) \\
& Q(k)=\sqrt{2 P} D(k) R[\rho(k)] \sin c\left[\pi T \Delta f_{d}(k)\right] \sin \left[\Delta f_{d}(k) k T+\varphi_{0}\right]+\tilde{n}_{Q}(k)
\end{aligned}
$$

The relevant power output:

$$
S(k)=\sqrt{I^{2}(k)+Q^{2}(k)}
$$

Found that when the code phase and carrier frequency capture, correlation power output to maximum.

As can be seen, the estimated residual code phase delay and Doppler frequency estimation residuals respectively correlation peak decreased $R[\rho(k)]$ and $\sin c\left[\pi T \Delta f_{d}(k)\right]$ times. If Doppler small changes, a quick operation that is able to traverse all the code phase to find a correlation peak, achieve capture PN code acquisition. But when the Doppler change is large, the correlation peak accumulate operations will produce a low-pass effect, seriously affecting the detection of the correlation peak.

Doppler shift estimation error and the correlation peak magnitude relationship as shown in Figure 2.

From Figure 2 it is clear that due to the cumulative effect in the relevant Summit Doppler detuning. When a single cumulative time of $0.1 \mathrm{~ms}$, its main lobe peak of the first cut-off frequency of $10 \mathrm{kHz}$; when the accumulated time is $0.2 \mathrm{~ms}$, the cut-off frequency of $5 \mathrm{kHz}$; when the accumulated time of $1 \mathrm{~ms}$, the correlation value of the frequency difference is $1 \mathrm{kHz}$ That time completely disappear. Although the increased accumulation time integral seen, the correlation peak can be increased to improve the detection probability of the correlation peak but increases with the frequency offset and the attenuation increases in speed, and therefore, the search for the maximum frequency step size will be reduced. The frequency search step size reduction, will lead to lower catch rate. In summary, the contradiction between integration time and frequency search step, so the actual capture, according to the specific application requirements eclectic background selection.

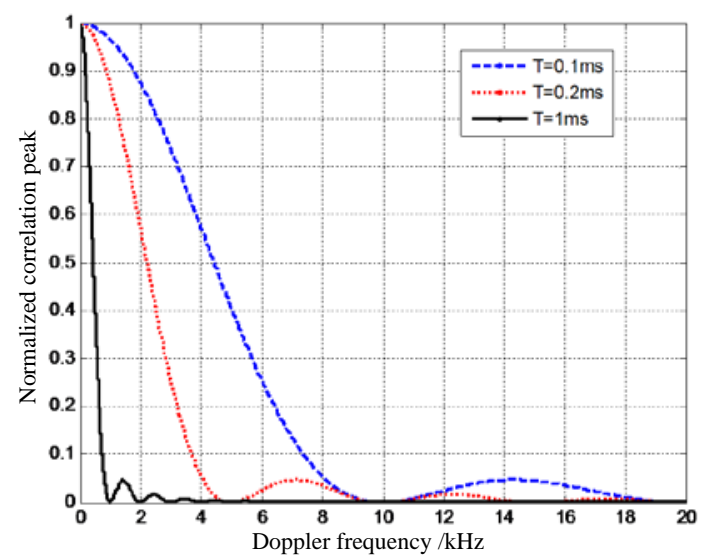

Figure 2. Doppler shift estimation error associated with peak amplitude relationship

In practical application, often to solve energy peak due to the Doppler detuning caused by declining issues by designing multi-channel parallel frequency bins. The frequency limit is generally divided groove $1 / 4$ cutoff frequency. Design principles shown in Figure 3.

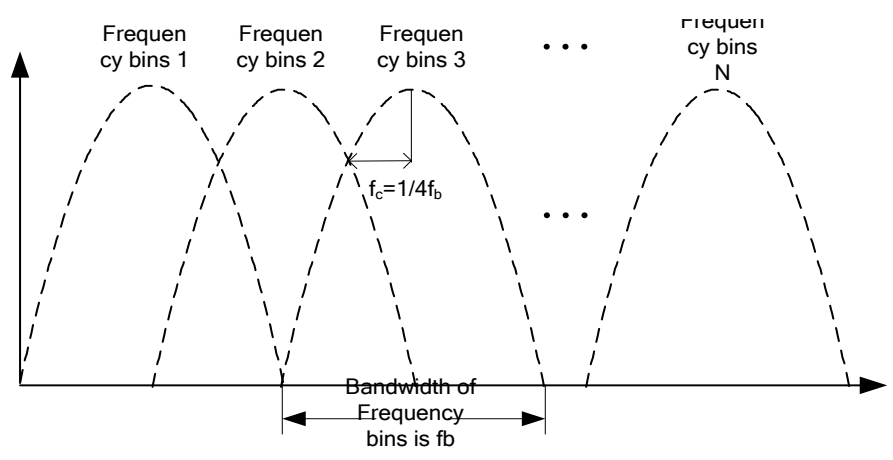

Figure 3. Schematic frequency dividing groove

Doppler frequency shift caused by the error can be offset by dividing the frequency bins. The signal frequency offset closest to the groove 3 , the highest correlation peak groove 3 , so you can quickly estimate code phase pseudo-code and approximate Doppler frequency range. 


\section{ZERO IMPACT ANALYSIS ON THE PERFORMANCE CAPTURE}

Pseudo code for a zero-fill cycle, could undermine the entire correlations pseudo-code sequence.

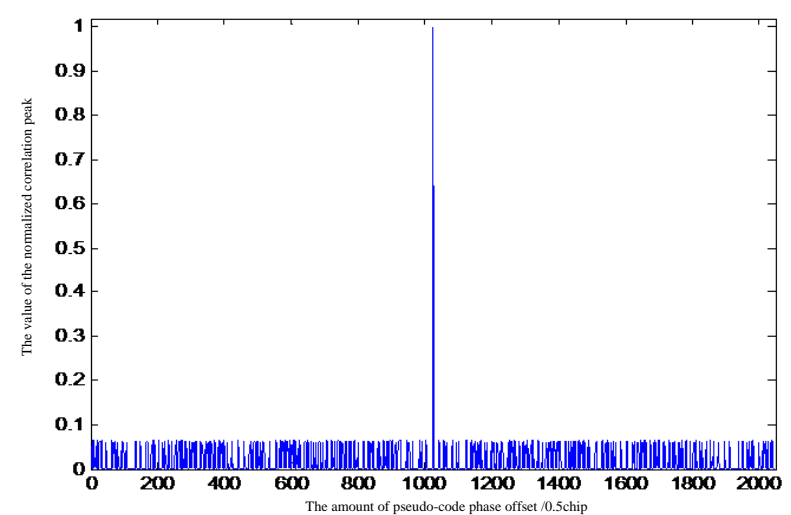

(a). Correlation peaks original sequence

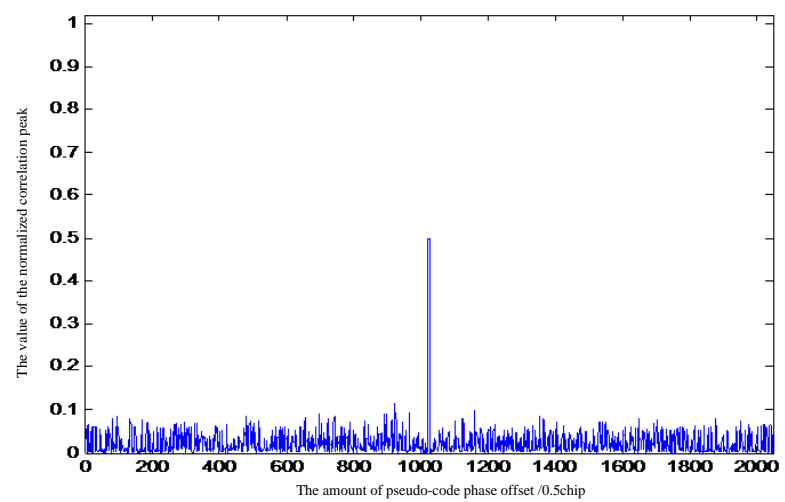

(b). A pseudorandom code period zeros after correlation peak

Figure 4. After a pseudorandom code period up zero correlation peak comparison

Process algorithm, since the local pseudo-code value is unchanged, output FFT conjugated do not change, you can advance through the computer to calculate the FFT conjugate results and stored in a storage device, and the FFT result of the received signal phase when multiplied, can be multiplied, do not do FFT conjugate. The FFT process twice and one IFFT converted into a FFT and one IFFT operation, thereby reducing the complexity of the algorithm, but also reduces the amount of computation to a certain extent, improve the capture rate.

\section{SUMMARY}

This chapter parallel acquisition algorithm based on cycle-related pseudo-code made in-depth discussions and simulation. Divided into derivation algorithm, the impact on the carrier search step acquisition performance, the impact of zeros on the acquisition performance analysis, to some extent, improve the capture rate. This approach in the case of Doppler forecast accuracy can be used to capture spread spectrum telemetry system.

\section{REFERENCES}

E.G.Strom, S.Parkvall, S.L.Miller and B.E.Ottersten.DSCDMA synchronization in time-varying fading channels[J]. IEEE Transactions on communications.2011.1636-1642.

E.G.Strom, S.Parkvall, S.L.Miller and B.E.Ottersten. Propagation delay estimation in asynchronous DS-CDMA systems[J]. IEEE Transactions on communications .2012:84-93.

M.G.El-tarhhuni and U.H.Sheikh.Performance analysis for an adaptive filter code-tracking technique in DS/SS systems[J]. IEEE Transactions on communications.2012:1058-1064.

U. Madhow. MMSE interference suppression fo timing acquisition and demodulation in DS-CDMA systems[J], IEEE Transactions on communications.2008:1065-1075.

Wang Shi lian, Zhang Chen, Gao Kai and zhang Er yang. Noncoherent PN code Acquisition Based on the Test of I/O LMS Adaptive Filters' Tap Weights[J]. IEEE Transactions on communications.2011:13-17. 